\title{
Autodeclaración del consumo de marihuana, tabaco, alcohol y derivados de cocaína en embarazadas en 2013 y 2016, Montevideo, Uruguay
}

\author{
Marijuana, tobacco, alcohol and cocaine use during pregnancy \\ in 2013 and 2016. A self-report study in Montevideo, Uruguay
}

\begin{abstract}
Mario Moraes Castro*, Fernanda Pinto**, Camila Pereiras**, Agustín Fischer**, Carolina Vogel**, Victoria Duarte**, José Barceló**, Claudio Sosa***, Gabriel González****.

* Prof. Agdo. Depto. de Neonatología, Centro Hospitalario Pereira Rossell, Facultad de Medicina. Universidad de la República, Montevideo. Uruguay. ** Centro Hospitalario Pereira Rossell, Facultad de Medicina. Universidad de la República, Montevideo. Uruguay. *** Prof. Agdo. de Ginecotocología, Clínica Ginecotocológica C, Centro Hospitalario Pereira Rossell, Facultad de Medicina. Universidad de la República, Montevideo. Uruguay. **** Prof. Dir. Cátedra de Neuropediatría, Centro Hospitalario Pereira Rossell, Facultad de Medicina. Universidad de la República, Montevideo. Uruguay.
\end{abstract}

\section{Resumen}

Introducción: Uruguay es el primer país en modificar la legislación sobre el consumo recreativo de cannabis, mediante la Ley 19.172 del 20 de diciembre de 2013, otorgando al Estado la potestad de regular su producción y comercialización. Objetivo: evaluar las prevalencias de la autodeclaración del consumo de marihuana, tabaco, alcohol, cocaína y derivados y su evolución temporal en embarazadas para los años 2013 y 2016. Metodología: estudio descriptivo, transversal y analítico, durante dos períodos de tiempo, en 2013 y 2016. Se incluyeron mujeres que tuvieron su parto y puerperio inmediato en el Centro Hospitalario Pereira Rossell (CHPR) de Montevideo, Uruguay. Se realizó una encuesta protocolizada sobre su nivel sociodemográfico, años de educación formal, datos del embarazo, parto, recién nacido y consumo de sustancias antes y durante la gestación. Resultados: en la investigación participaron 577 mujeres, 319 correspondientes a las entrevistas realizadas en el año 2013 y 258 en 2016. Respecto al alcohol, $76(23,82 \%)$ mujeres declaran su consumo durante el embarazo en 2013, mientras que, en 2016, $91 \quad(35,3 \%)(p=0,003)$. En cuanto al tabaco, 59 (43\%) dejaron de fumar al enterarse del embarazo en 2013 y $69(60 \%)$ en 2016 ( $\mathrm{p}=0,008)$. En 2013, 41 mujeres $(12,85 \%)$ fumaron marihuana hasta saber del embarazo y $79(30 \%)$ en 2016 $(\mathrm{p}<0,001)$. Durante la gestación, en 2013 declararon fumar marihuana 5 mujeres $(1,57 \%)$, mientras que en 2016 fueron 28 (10,85\%) (p<0,001). El uso de cocaína y pasta base de cocaína (PBC) no cambió significativamente entre 2013 y 2016. Conclusiones: Entre los años 2013 y 2016 se observó un aumento significativo en la autodeclaración del consumo de marihuana y alcohol antes y durante el embarazo. El consumo de cocaína y pasta base de cocaína se mantuvo estable en ese periodo. El consumo de tabaco disminuyó significativamente en mujeres embarazadas.

Palabrasclave:Cannabis; Tabaco; Alcohol;Embarazo; Autodeclaración.

\section{Abstract}

Background: Uruguay passed Law 19.172 on December 20, 2013, thus enabling the State to regulate the production, distribution, sale, and consumption of cannabis. Objectives: to determine the change in maternal self-report of marijuana, tobacco, alcohol, and cocaine use during pregnancy between 2013 and 2016. Methods: cross-sectional, descriptive, analytical study between 2013 and 2016. The sample consists of pregnant woman who delivered and received immediate postpartum care at the Centro Hospitalario Pereira Rossell in Montevideo, Uruguay. The instrument was a protocolized survey that assessed outcomes such as sociodemographic level, years of formal education, data on pregnancy, childbirth, newborn, and substance use before and during pregnancy. Results: the sample consisted of 577 pregnant women, 319 corresponding to interviews conducted in 2013 and 258 in 2016. Regarding alcohol intake, 76 (23.82\%) women reported drinking while pregnant in 2013, whereas in 2016, $91(35,3 \%)$ $(p=.003)$ did so. As for tobacco, 59 (43\%) quit smoking when they found out about their pregnancy in 2013, and 69 (60\%) quit in 2016 $(\mathrm{p}=0,008)$. In 2013, 41 women $(12.85 \%)$ smoked marijuana until they discovered their pregnancy, and $79(30 \%)$ did so in $2016(p<.001)$. The use of cocaine and its derivatives did not change significantly between 2013 and 2016. Conclusions: between 2013 and 2016, there was a significant increase in the self-report of marijuana and alcohol consumption before and during pregnancy. The consumption of cocaine and cocaine base paste remained stable during this period. Tobacco use decreased significantly in pregnant women. Key Words: Cannabis; Tobacco; Alcohol; Pregnancy; Self-report. 
$\mathrm{E}$ n Uruguay se está llevando a cabo un cambio en la legislación sobre el consumo de sustancias de uso recreativo específicamente tabaco, alcohol y cannabis. En 2008 se aprobó la Ley 18.256 que regula la comercialización del tabaco y su consumo en espacios públicos. Esta ley prohíbe fumar o mantener encendidos productos de tabaco en espacios cerrados públicos o privados y toda forma de publicidad, promoción y patrocinio de los productos de tabaco. Actualmente existen líneas de trabajo para reglamentar el consumo de bebidas alcohólicas. Uruguay es el primer país en modificar la legislación sobre el consumo recreativo de cannabis, mediante la Ley 19.172, otorgando al Estado la potestad de regular su producción y comercialización, permitiendo la plantación, el cultivo y la venta de cannabis psicoactivo, bajo la supervisión de los organismos reguladores del Estado uruguayo. El consumo de sustancias de uso recreativo legales e ilegales durante el embarazo genera un impacto negativo en la salud de la mujer y el desarrollo fetal (Ko, Farr, Tong, Creanga y Callaghan, 2015). Es importante conocer las características del consumo en la población para orientar políticas sanitarias con el objetivo de estimular conductas saludables.

Según la Junta Nacional de Drogas de Uruguay, el $62,2 \%$ de las mujeres uruguayas consumieron bebidas alcohólicas en el último año, encontrándose su pico de incidencia entre los 26 y 35 años, período que coincide con la edad sexual reproductiva de la mujer (Junta Nacional de Drogas Uruguay, 2016). Esta conducta durante el embarazo determina un aumento de la probabilidad de aparición de Síndrome de Alcohol Fetal (FAS) y sus alteraciones en el desarrollo, persistentes durante toda la vida, agrupadas bajo la denominación Espectro de Alteraciones por Alcohol Fetal (Valentine et al., 2017; Myers et al., 2017).

Si bien la prevalencia de tabaquismo en Uruguay descendió a consecuencia de la implementación de leyes antitabaco, 24,9\% de las mujeres uruguayas son fumadoras. El tabaquismo tanto activo como pasivo durante la gestación determina un aumento en la prevalencia de complicaciones materno-fetales como lo son el bajo peso al nacer y Síndrome de Muerte Súbita del Lactante (SMSL) (Berrueta et al., 2016; Cooper et al., 2017).

A nivel mundial se asiste un incremento en el consumo de marihuana con fines recreativos, con una mayor aceptación social y cambios legislativos que lo regulan. La exposición a cannabis durante el embarazo aumenta el riesgo de anemia en la mujer y en el recién nacido probabilidad de ingreso a Unidad de Cuidados Intensivos Neonatales (Emery, Gregory, Grace y Levine, 2016; Gunn et al., 2016). El consumo de cannabis en el embarazo no determina defectos congénitos y no se reconoce al momento actual un fenotipo característico (Warner, Roussos-Ross y Behnke, 2014). Se asocia con una disminución del peso al nacer y del perímetro cefálico. Esta alteración del crecimiento fetal es dosis dependiente (Conner et al., 2015). No se observó relación entre el consumo de marihuana durante el embarazo y el coeficiente intelectual, pero existe afectación en las funciones ejecutivas como la atención, memoria de corto plazo y procesamiento verbal (Goldschmidt, Richardson, Larkby y Day, 2016; Goldschmidt, Richardson, Willford y Day, 2008). Estas alteraciones pueden estar relacionadas con cambios anatómicos en la corteza cerebral descritos en resonancia magnética nuclear (El Marroun et al., 2016; Grant, Petroff, Isoherranen, Stella y Burbacher, 2017).

El consumo de cocaína y sus derivados tiene una marcada repercusión en la salud materna y fetal, además de consecuencias médico-legales, por lo que es fundamental identificar aquellos casos de consumo para un correcto seguimiento del recién nacido expuesto (García, Campistol, López-Vilchez, Morcillo y Mur, 2017). Su consumo provoca hipertensión materna, vasoconstricción y disminución del flujo uterino, determinando un menor aporte de oxígeno y nutrientes al feto. Aumenta el riesgo de prematurez y muerte intrauterina. Se ha descrito mayor incidencia de neuroexcitabilidad pero no se ha demostrado la presencia de un síndrome de abstinencia claro. En cuanto al crecimiento, se asocia a menor peso al nacer, longitud y perímetro cefálico. Durante la niñez se observan alteraciones en el coeficiente intelectual y atención, con mayor incidencia de déficits atencionales e hiperactividad (Smith y Santos, 2016). En Uruguay el consumo y tenencia de sustancias psicoactivas para uso personal no es penado por la ley.

Es relevante conocer la evolución de la autodeclaración del consumo de sustancias de uso recreativo en embarazadas, en el contexto de cambios en políticas sanitarias. El consumo de sustancias psicoactivas tiene efectos negativos para la salud de la madre y de sus hijos. Las conductas de consumo de sustancias y actos negligentes por parte de la embarazada pueden vulnerar los derechos de sus hijos. Es importante evaluar como los cambios en las políticas sanitarias pueden influir de forma positiva o negativa en el consumo de sustancias en embarazadas.

El objetivo del presente estudio es evaluar las prevalencias de la autodeclaración del consumo de marihuana, tabaco, alcohol, cocaína y derivados y su evolución temporal para los años 2013 y 2016.

\section{Material y métodos}

Se realizó un estudio descriptivo, transversal y analítico durante dos periodos de tiempo, en 2013 y 2016. Se incluyeron mujeres que tuvieron su parto y puerperio inmediato en el Centro Hospitalario Pereira Rossell (CHPR) de Montevideo, Uruguay. El CHPR es un hospital público de referencia para Montevideo y área metropolitana en la asistencia del niño, la mujer y la embarazada, cuya población característicamente proviene de los sectores 
más vulnerables de la sociedad. El tamaño muestral fue estimado tomando como referencia la sustancia de menor consumo esperado, cocaína y sus derivados. A partir de dicho cálculo se obtuvo un número de casos a incluir de 252 mujeres, para una población de 8000 partos anuales en el CHPR con una proporción esperada de $6,5 \%$ con un nivel de confianza de $95 \%$ y una precisión de $3 \%$ (rango 3,5\%-9,5\%) (Moraes, Sosa, Umpiérrez y González, 2014). El tamaño muestral calculado y el método de aleatorización tienen como propósito garantizar la representatividad de la muestra.

Los criterios de exclusión determinados fueron: recién nacido menor de 35 semanas de edad gestacional, embarazo múltiple, infección congénita del sistema nervioso central, malformaciones congénitas mayores y recién nacido expuesto a infección por Virus de Inmunodeficiencia Humana (VIH) o con bajo peso al nacer.

Como estos factores influyen negativamente sobre el desarrollo infantil a largo plazo y los autores consideran en una segunda etapa evaluar los efectos de las sustancias de consumo sobre el mismo, se optó por estos criterios de exclusión.

Para que la muestra fuera representativa de toda la población se realizó un muestreo probabilístico mediante el diseño de un programa informático de aleatorización que en base al número de nacimientos de las últimas 24 horas seleccionaba el caso de estudio. En el caso de que la participante debiera ser excluida, o que no aceptara el consentimiento informado, el programa asignaba un caso alternativo.

Luego de obtener el consentimiento informado del caso seleccionado, se realizó una encuesta protocolizada sobre su nivel sociodemográfico, datos del embarazo, parto, recién nacido y consumo de sustancias antes y durante la gestación. Posteriormente, se analizó su historia clínica para corroborar dicha información.

La encuesta fue estructurada con un diseño claro, sin ambigüedades. Previo al inicio del estudio en 2013 y en 2016 se realizó un estudio piloto entrevistando a 20 mujeres en cada año, con el objetivo de reconocer sus debilidades y fortalezas, luego de lo cual el equipo de trabajo validó el formulario definitivo.

Las entrevistas fueron realizadas por cuatro miembros del equipo de investigación previamente entrenados para dicho fin. Cada participante se identificó con un número de tres cifras correlativo en el momento de la entrevista. De esta manera no se solicitaba información personal y se mantenía la confidencialidad durante todo el proceso.

Se recabó la siguiente información sociodemográfica: nombre materno, fecha de nacimiento materna, etnia, estado civil, convivencia con su pareja, nivel educativo, trabajo, composición del hogar, ingresos, planificación del embarazo. Para la categorización de los ingresos se utilizan las definiciones del Instituto Nacional de Estadística (INE) de Uruguay en donde se define en situación de indigencia a aquellos hogares cuyos ingresos no pueden satisfacer sus necesidades básicas de alimentación.

En cuanto a los consumos de marihuana, tabaco, alcohol, cocaína y pasta base de cocaína (PBC) las preguntas incluían: ¿Alguna vez ha fumado/tomado?, ¿cuántos años?, ¿cuántos por día?, ¿por mes?, ¿cuántos después de saber que estaba embarazada?, ¿su compañero consume?, ¿consume en la misma habitación?, ¿cuándo dejó de consumir? Para este estudio se considera consumo activo cualquier tipo de consumo durante el embarazo (consumo sin saber que estaba embarazada y consumo luego de saber que estaba embarazada).

Del parto y del recién nacido: vía de nacimiento, sexo, peso al nacer, APGAR, edad gestacional, lugar de internación, características del examen físico.

Los datos se cargaron en una base de datos de ACCESS ${ }^{\circledR}$ y fueron posteriormente analizados en el paquete estadístico STATA v11. En primera instancia se realizó un análisis univariado con el objetivo de reconocer los valores que estuvieran fuera de rango. En caso de estarlo, el investigador principal comparaba dichos datos con la encuesta original para poder validarlos o corregirlos.

Las variables cualitativas (edad, estado civil, nivel de ingreso familiar, nivel educativo) se presentan en números absolutos y en los porcentajes correspondientes, se usa la prueba chi-cuadrado como contraste de hipótesis. En todos los casos, se consideró el mínimo nivel de significación $\mathrm{p}<0,05$.

La identificación personal de cada individuo fue confidencial, no siendo posible su identificación en el reporte ni tampoco en las publicaciones. Los cuestionarios realizados fueron eliminados tras su incorporación a la base de datos y su validación posterior.

El presente estudio fue aprobado por el Comité de Ética del Centro Hospitalario Pereira Rossell previo a su inicio.

\section{Resultados}

En la investigación participaron 577 mujeres, 319 correspondientes a las entrevistas realizadas en el año 2013 y 258 en 2016.

Las características sociodemográficas de la muestra se resumen en la tabla 1. A excepción de la variable nivel de ingreso familiar, no se hallaron diferencias estadísticamente significativas entre ambos grupos. Del grupo entrevistado en 2013 se destaca que del total (319), 267 eran mayores de 18 años (83,65\%), con una media de 24,67 años, con un máximo de edad de 42 y mínimo de 13 años. En la variable educación, 123 mujeres $(38,56 \%)$ cursaron menos de seis años de estudio. Para el total de entrevistadas en 2016 (258), 210 eran mayores de 18 años (86,82\%), con una media de 24,09 años, apreciándose un máximo y mínimo de edad de 42 y 13 años respectivamente. En referen- 
Tabla 1. Características sociodemográficas de la muestra en los años $2013(N=319)$ y $2016(N=258)$.

\begin{tabular}{|c|c|c|c|}
\hline Datos sociodemográficos & $\begin{array}{c}\text { Valores N (\%) } \\
2013\end{array}$ & $\begin{array}{c}\text { Valores N (\%) } \\
2016\end{array}$ & Valor $p$ \\
\hline Edad & & & 0,214 \\
\hline 18 o menor & $52(16,3)$ & $34(13,8)$ & \\
\hline $19-34$ & $241(75,5)$ & $210(81,4)$ & \\
\hline 35 o mayor & $26(8,15)$ & $14(5,4)$ & \\
\hline Estado civil & & & 0,371 \\
\hline Casada & $34(10,66)$ & $17(6,6)$ & \\
\hline Vive con su pareja ${ }^{1}$ & $184(57,68)$ & $151(58,5)$ & \\
\hline Soltera & $98(30,7)$ & $87(33,7)$ & \\
\hline Divorciada & $3(0,94)$ & $2(0,78)$ & \\
\hline Sin Dato/No contesta & 0 & $1(0,39)$ & \\
\hline Nivel educativo & & & 0,658 \\
\hline Ninguno & 0 & $1(0,39)$ & \\
\hline Primaria ${ }^{2}$ & $123(38,56)$ & $91(35,3)$ & \\
\hline Secundaria ${ }^{3}$ - UTU 4 & $188(58,93)$ & $160(62)$ & \\
\hline Terciaria & $8(2,51)$ & $5(1,94)$ & \\
\hline Sin Dato/No contesta & 0 & $1(0,39)$ & \\
\hline Planificación del embarazo & & & 0,360 \\
\hline $\mathrm{Si}$ & $137(42,95)$ & $101(39,1)$ & \\
\hline No & $181(56,74)$ & $156(60,5)$ & \\
\hline Sin Dato/No contesta & $1(0,31)$ & $1(0,31)$ & \\
\hline Vive con su pareja & & & 0,970 \\
\hline No & $79(24,7)$ & $64(24,8)$ & \\
\hline $\mathrm{Si}$ & $240(75,24)$ & $193(0,75)$ & \\
\hline Sin determinar & 0 & $1(0,39)$ & \\
\hline Nivel de ingreso familiar 5 & & & 0,026 \\
\hline Indigencia & $152(47,8)$ & $110(42,6)$ & \\
\hline Pobreza & $142(44,65)$ & $134(52)$ & \\
\hline Sobre línea de pobreza & $18(5,66)$ & $5(1,94)$ & \\
\hline No contesta & $7(2,19)$ & $9(2,7)$ & \\
\hline
\end{tabular}

Nota. ${ }^{1}$ Más de un año viviendo con la misma pareja.

${ }^{2}$ Educación primaria, primeros 6 años de estudio.

${ }^{3}$ Educación secundaria, 6 a 12 años de estudio.

4 Universidad del Trabajo del Uruguay, entre 9 y 12 años de estudio.

${ }^{5}$ Nivel de ingresos por escala del Instituto Nacional de Estadística y Censo del Uruguay.

cia al nivel socioeconómico, 18 mujeres (5,6\%) en 2013 se encontraban por encima de la línea de pobreza y 157 (47\%) declararon niveles de ingreso familiar en el rango de indigencia, la ocupación más frecuente del jefe de familia $(60,8 \%)$ fue el trabajo informal por breves períodos de tiempo. En cuanto a los datos de 2016, 5 participantes manifestaron ingresos por encima del nivel de pobreza $(1,94 \%)$ y $110(42,6 \%)$ declararon niveles de indigencia. Estas diferencias entre las encuestas de 2013 y 2016 son estadísticamente significativas. El embarazo no planificado fue la situación más frecuente en ambos períodos, 182 casos $(57 \%)$ en 2013 y $156(60,5 \%)$ en 2016.

La tabla 2 resume las conductas de consumo de tabaco y alcohol durante el embarazo.

Tabla 2. Diferencias en la frecuencia del consumo de drogas por autodeclaración en mujeres embarazadas, entre el 2013 y 2016.

\begin{tabular}{llll}
\hline Drogas por autodeclaración & $\begin{array}{c}\text { Valores N (\%) } \\
\mathbf{2 0 1 3}\end{array}$ & $\begin{array}{c}\text { Valores N (\%) } \\
\mathbf{2 0 1 6}\end{array}$ & Valor p \\
\hline Consumo activo de tabaco* & & & 0,611 \\
\hline $\mathrm{Si}$ & $136(42,63)$ & $115(44,6)$ & \\
No & $183(57,37)$ & $142(55)$ & \\
Sin Dato/No contesta & 0 & $1(0,39)$ & \\
\hline Exposición pasiva al tabaco & & & 0,000 \\
\hline Si & $167(52,35)$ & $62(24)$ & \\
No & $152(47,65)$ & $196(52,7)$ & \\
\hline Consumo de alcohol & & & 0,003 \\
\hline Si & $76(23,82)$ & $91(35,3)$ & \\
No & $241(75,54)$ & $166(64,3)$ & \\
Sin Dato/No contesta & $2(0,63)$ & $1(0,39)$ & \\
\hline Embriaguez & & & 0,235 \\
\hline Si & $7(2,19)$ & $10(3,8)$ & \\
No & $312(97,78)$ & $248(96,2)$ & \\
\hline
\end{tabular}

Nota. *Incluye cualquier tipo de consumo durante el embarazo (consumo sin saber que estaba embarazada y consumo luego de saber que estaba embarazada).

Respecto al alcohol, 76 (23,82\%) mujeres declaran su consumo durante el embarazo en 2013, mientras que 91 (35,3\%) de ellas lo reportan en 2016. Estas diferencias son estadísticamente significativas. El número de episodios de embriaguez no varía significativamente en ambos períodos de la investigación.

En 2013, 189 mujeres fumaron alguna vez en su vida, 53 (28\%) dejaron de fumar antes del embarazo y 136 mujeres $(42,63 \%)$ fumaron en alguna ocasión durante el embarazo. En 2016, 143 mujeres fumaron alguna vez en su vida, 28 (20\%) abandonaron el hábito previo a la gestación y 115 $(44,6 \%)$ fumaron en alguna ocasión durante el embarazo.

De aquellas que fumaban tabaco activamente al momento del embarazo, 59 (43\%) dejaron de hacerlo al enterarse del mismo según las encuestas del 2013, aumentando dicha cifra en 2016 a $69(60 \%)(\mathrm{p}=0,008)$. De las mujeres que continuaron fumando, $52(38 \%)$ y $35(30,4 \%)$ fumaron, pero en menor cantidad para 2013 y 2016 respectivamente. Por último, 25 (18\%) y $11(9,5 \%)$, respectivamente en 2013 y 2016, continuaron su hábito tabáquico sin modi- 
ficación alguna. Estas diferencias fueron estadísticamente significativas (tabla 3).

Tabla 3. Diferencias en la frecuencia del consumo de tabaco en mujeres antes y durante el embarazo, entre el 2013 y 2016.

\begin{tabular}{llll}
\hline Consumo de tabaco & $\begin{array}{c}\text { Valores N (\%) } \\
\mathbf{2 0 1 3}\end{array}$ & $\begin{array}{l}\text { Valores N (\%) } \\
\mathbf{2 0 1 6}\end{array}$ & Valor $\mathbf{~}$ \\
\hline Fumaron alguna vez en su vida & & & 0,365 \\
\hline $\mathrm{Si}$ & $189(59,2)$ & $143(55,9)$ & \\
No & $130(40,8)$ & $115(44,1)$ & \\
Total & $319(100)$ & $258(100)$ & \\
\hline Dejaron de fumar antes del embarazo & & & 0,075 \\
\hline Si & $53(28)$ & $28(20)$ & \\
No & $136(72)$ & $115(80)$ & \\
Total & $189(100)$ & $143(100)$ & \\
\hline Fumaron en el embarazo & & & 0,020 \\
\hline Fumaron hasta saber & $59(43)$ & $69(60)$ & \\
Fumaron en menor cantidad & $52(38)$ & $35(30,4)$ & \\
Continuaron fumando igual que & $25(18)$ & $11(9,5)$ & \\
antes & $136(100)$ & $115(100)$ & \\
\hline Total & &
\end{tabular}

Con respecto al consumo de marihuana, se destaca un aumento en la declaración de su consumo de un período a otro. Durante la gestación, en 2013 declararon fumar marihuana 5 mujeres $(1,57 \%)$, mientras que en 2016 fueron $28(10,85 \%)(\mathrm{p}<0,001)$. En 2013, 41 mujeres $(12,85 \%) \mathrm{fu}-$ maron marihuana hasta saber del embarazo y $79(30 \%)$ en $2016(\mathrm{p}<0,001)$. Fumaron marihuana alguna vez en la vida $46(14,4 \%)$ en 2013 y $107(41,4 \%)$ en 2016 (p<0,001). La frecuencia de consumo se muestra en la tabla 4 .

El uso de cocaína y PBC no cambió significativamente entre 2013 y 2016. En 2013 consumieron cocaína 8 (2,5\%) mujeres y en 2016 fueron $8(3,1 \%)$.

\section{Discusión}

La sustancia que más frecuentemente utilizaron las mujeres durante el embarazo según autodeclaración en ambos períodos del estudio fue el tabaco, con una frecuencia del 39,9\% en 2016. Se observó un descenso significativo del consumo de tabaco durante la gestación entre 2013 y 2016 ( $\mathrm{p}=0,02$ ). El número de embarazadas expuestas pasivamente al humo de tabaco disminuyó en forma significativa $(p<0,001)$. La frecuencia de abandono del hábito de fumar al enterarse del embarazo fue de $43 \%$ en 2013 y de $60 \%$ en 2016. El embarazo es probablemente el evento que

Tabla 4. Diferencias en la frecuencia del consumo de marihuana, cocaína y pasta base de cocaína (PBC) en mujeres antes y durante el embarazo, entre el 2013 y 2016.

\begin{tabular}{|c|c|c|c|c|c|c|}
\hline \multirow[b]{2}{*}{ Marihuana } & \multicolumn{3}{|c|}{ Valores N 2013} & \multicolumn{3}{|c|}{ Valores N 2016} \\
\hline & Antes ${ }^{\star} \Omega$ & Durante $¥$ & Total $\Omega$ & Antes ${ }^{\star} \Omega$ & Durante $¥$ & Total $\Omega$ \\
\hline Experimental & 2 & 0 & 2 & 32 & 0 & 32 \\
\hline Mensual & 18 & 2 & 20 & 11 & 2 & 13 \\
\hline Semanal & 12 & 1 & 13 & 22 & 8 & 30 \\
\hline Total & 41 & 5 & 46 & 79 & 28 & 107 \\
\hline Cocaína & Antes* & Durante $¥$ & Total & Antes* & Durante $¥$ & Total \\
\hline Experimental & 0 & 0 & 0 & 0 & 0 & 0 \\
\hline Mensual & 1 & 1 & 2 & 1 & 2 & 3 \\
\hline Semanal & 1 & 1 & 2 & 5 & 0 & 5 \\
\hline Diario & 3 & 1 & 4 & 0 & 0 & 0 \\
\hline Total & 5 & 3 & 8 & 6 & 2 & 8 \\
\hline PBC & Antes* & Durante $¥$ & Total & Antes* & Durante $¥$ & Total \\
\hline Experimental & 0 & 0 & 0 & 0 & 0 & 0 \\
\hline Mensual & 1 & 2 & 3 & 2 & 0 & 2 \\
\hline Semanal & 1 & 1 & 2 & 0 & 1 & 1 \\
\hline Diario & 5 & 1 & 6 & 4 & 0 & 4 \\
\hline Total & 7 & 4 & 11 & 6 & 1 & 7 \\
\hline
\end{tabular}

Nota. Los datos están expresados en frecuencia absoluta (N).

* Refiere a consumo antes del embarazo o su abandono al enterarse del embarazo.

$¥$ Refiere a consumo durante el embarazo.

$\Omega$ Valor p<o,001 para el contraste entre 2013 y 2016. 
más motiva a dejar de fumar y la campaña nacional antitabaco que comenzó con los cambios de legislación en 2008 provee un marco ideal para el abandono del hábito tabáquico. En un estudio realizado en Reino Unido se observó que la motivación para el cambio de hábitos de consumo de sustancias durante el embarazo disminuye significativamente mientras el embarazo progresa. Hasta un tercio de las mujeres que no fumaron en el embarazo volvieron a fumar en los tres meses posteriores al parto (Cooper et al., 2017). El tabaco sigue siendo en el Uruguay, una sustancia legal y de fácil acceso. Estos datos afirman la necesidad de intervenciones reiteradas y persistentes en el tiempo para lograr cambios permanentes en los hábitos de consumo.

Con respecto a la ingesta de bebidas alcohólicas durante el embarazo, se observó un aumento del 23,8\% en 2013 a un 35,3\% en 2016 ( $\mathrm{p}=0,003$ ). Desconocemos si este fenómeno pudiera ser explicado por un efecto de complementación (en donde las políticas más liberales sobre el consumo de marihuana producen un aumento complementario del consumo de alcohol), producto de la reciente legislación del consumo recreativo de cannabis (Guttmannova et al., 2016). Si bien actualmente se está avanzando en la regulación de la venta de alcohol, su consumo no se encuentra regulado. El alcohol actualmente es la sustancia más consumida alguna vez en la vida en la población general en Uruguay, según una encuesta realizada por la Junta Nacional de Drogas, siendo también considerada como la que causa mayor consumo problemático (Junta Nacional de Drogas Uruguay, 2016).

Se observa un aumento en la declaración del consumo de marihuana alguna vez en la vida, de $14,4 \%$ a $41,4 \%$ entre los años 2013 a 2016. El Reporte Mundial de consumo de drogas en 2014 refiere que la frecuencia de consumo de marihuana es de 12,4\% en África, 12,1\% en Norteamérica y $15,2 \%$ en Colombia (United Nations Office on Drugs and Crime, 2014). Durante la gestación, en 2013 declararon fumar marihuana $1,57 \%$ de las mujeres, mientras que en 2016 el 10,85\%. Este aumento fue estadísticamente significativo $(p<0,001)$. El embarazo es uno de los momentos clave para la realización de cambios en el estilo de vida maternos. El momento vital en el cual la mujer se embaraza puede ser un factor determinante de las características del consumo de marihuana (De Genna, Cornelius, Goldschmidt y Day, 2015; Elder, 1975). Las intervenciones realizadas durante la gestación pueden tener un impacto positivo en la disminución del consumo de sustancias.

La droga con menor consumo en la población de estudio fue cocaína. Su consumo es importante por los efectos que tiene sobre la madre, el embarazo y el recién nacido. No se observa una variación significativa en la autodeclaración del consumo de esta sustancia.

El consumo de sustancias en el embarazo tiene implicancias no solo biológicas, sobre la madre y el feto, sino sociales y legales. No todos los países han decidido abordar la problemática de la misma manera. En Gales, el proyecto de ley "Zoe's Law" pretendió otorgar personería jurídica a aquellos fetos mayores de 20 semanas lo que permitiría responsabilizar a las madres por actos negligentes y a los proveedores de salud a priorizar, en algunos casos el bienestar fetal en contra de los deseos de la madre. Otros países optan por la inclusión de la madre en un programa de tratamiento de adicciones (Hotham, Ali, White y Robinson, 2016). En España, la implementación progresiva de leyes antitabaco que establecían la prohibición de fumar en espacios públicos en dos fases, una parcial y una más restrictiva, se asoció a una reducción del riesgo de parto de pretérmino $(4,5 \%)$ y de bajo peso al nacer $(2,3 \%)$. Este beneficio para la salud fue especialmente evidente con la introducción de la prohibición más restrictiva (Simon et al., 2017). En Uruguay se implementó en 2008 la Ley 18.256 con este mismo fin.

Los cambios macroeconómicos que se observaron en Uruguay entre 2013 y 2016 han determinado cambios en el nivel de ingreso familiar en la población de estudio. El consumo de sustancias es más frecuente en las poblaciones con menor nivel de ingreso familiar. Estos cambios no explican el aumento de la autodeclaración de consumo observado para marihuana.

Existen algunas limitaciones en el estudio por utilizar la autodeclaración como método de identificación. La negación o subdeclaración de aquellas sustancias que son percibidas como potencialmente nocivas o sin aceptación social determina una cifra menor de reporte. No obstante, la aplicación de un mismo cuestionario en los dos períodos de tiempo, 2013 y 2016, nos permite observar cambios evolutivos en los hábitos de consumo. Otra limitante es la exclusión de recién nacidos menores de 35 semanas de edad gestacional que puede no considerar a aquellas mujeres que tuvieron un parto prematuro debido al uso excesivo de sustancias nocivas. La población participante del trabajo de investigación proviene de los sectores más vulnerables de la sociedad uruguaya, con un elevado nivel de indigencia, pobreza y embarazo no planificado. Por ello, estos resultados no pueden ser extrapolados a la población general o a otros contextos socioculturales. Por último, dado que las mismas personas que pasan los cuestionarios son miembros del equipo de investigación podría existir sesgo del entrevistador.

\section{Conclusiones}

Entre los años 2013 y 2016 se observó un aumento significativo en la autodeclaración del consumo de marihuana y alcohol antes y durante el embarazo. El consumo de cocaína y PBC se mantuvo estable en ese periodo. El consumo de tabaco disminuyó significativamente en mujeres embarazadas. El diseño de la investigación no permite determinar si este dato se relaciona con un aumento del consumo 
o de su autodeclaración al percibirse un ambiente de mayor tolerancia social y legal.

\section{Financiación}

La financiación de este estudio fue proporcionada por el Espacio Interdisciplinario de la Universidad de la República y por el Fondo Sectorial Salud en la Primera Infancia de la Agencia Nacional de Investigación e Innovación (ANII).

\section{Conflicto de Intereses}

Los autores declaran no tener ningún conflicto de intereses.

\section{Bibliografía}

Berrueta, M., Morello, P., Alemán, A., Tong, V. T., Johnson, C., Dietz, P. M., ... Althabe, F. (2016). Smoking Patterns and Receipt of Cessation Services Among Pregnant Women in Argentina and Uruguay. Nicotine $\mathcal{E}^{2}$ Tobacco Research, 18, 1116-1125. doi:10.1093/ntr/ntv145.

Conner, S. N., Carter, E. B., Tuuli, M. G., MacOnes, G. A. y Cahill, A. G. (2015). Maternal marijuana use and neonatal morbidity. American Journal of Obstetrics and Gynecology, 213, 422e1-422e4. doi:10.1016/j.ajog.2015.05.050.

Cooper, S., Orton, S., Leonardi-Bee, J., Brotherton, E., Vanderbloemen, L., Bowker, K., ... Coleman, T. (2017). Smoking and quit attempts during pregnancy and postpartum: a longitudinal UK cohort. BMJ Open, 7, e018746. doi:10.1136/bmjopen-2017-018746.

De Genna, N. M., Cornelius, M. D., Goldschmidt, L. y Day, N. L. (2015). Maternal age and trajectories of cannabis use. Drug and Alcohol Dependence, 156, 199-206. doi:10.1016/j.drugalcdep.2015.09.014.

El Marroun, H., Tiemeier, H., Franken, I. H. A., Jaddoe, V. W. V., Van der Lugt, A., Verhulst, F. C., ... White, T. (2016). Prenatal Cannabis and Tobacco Exposure in Relation to Brain Morphology: A Prospective Neuroimaging Study in Young Children. Biological Psychiatry, 79, 971-979. doi:10.1016/j.biopsych.2015.08.024.

Elder, G. H. (1975). Age differentiation and the life course. Annual Review of Sociology, 1, 165-190.

Emery, R. L., Gregory, M. P., Grace, J. L. y Levine, M. D. (2016). Prevalence and correlates of a lifetime cannabis use disorder among pregnant former tobacco smokers. Addictive Behaviors, 54, 52-58. doi:10.1016/j. addbeh.2015.12.008.

Garcia, J., Campistol, E., López-Vilchez, M. Á., Morcillo, M. J. y Mur, A. (2018). Análisis del maltrato prenatal en Cataluña entre los años 2011 y 2014. Anales de Pediatría, 88, 150-159. doi:10.1016/j.anpedi.2017.04.011.
Goldschmidt, L., Richardson, G. A., Willford, J. y Day, N. L. (2008). Prenatal marijuana exposure and intelligence test performance at age 6. Journal of the American Academy of Child and Adolescent Psychiatry, 47, 254-263. doi:10.1097/CHI.0b013e318160b3f0.

Goldschmidt, L., Richardson, G. A., Larkby, C. y Day, N. L. (2016). Early marijuana initiation: The link between prenatal marijuana exposure, early childhood behavior, and negative adult roles. Neurotoxicology and Teratology, 58, 40-45. doi:10.1016/j.ntt.2016.05.011.

Grant, K. S., Petroff, R., Isoherranen, N., Stella, N. y Burbacher, T. M. (2017). Cannabis use during pregnancy: Pharmacokinetics and effects on child development. Pharmacology EO Therapeutics, 182, 133-151. doi:10.1016/j. pharmthera.2017.08.014.

Gunn, J. K. L., Rosales, C. B., Center, K. E., Nuñez, A., Gibson, S. J., Christ, C. y Ehiri, J. E. (2016). Prenatal exposure to cannabis and maternal and child health outcomes: a systematic review and meta-analysis. BMJ Open, 6, e009986. doi:10.1136/bmjopen-2015-009986.

Guttmannova, K., Lee, C. M., Kilmer, J. R., Fleming, C. B., Rhew, I. C., Kosterman, R. y Larimer, M. E. (2016). Impacts of Changing Marijuana Policies on Alcohol Use in the United States. Alcoholism: Clinical and Experimental Research, 40, 33-46. doi:10.1111/acer.12942.

Hotham, E. D., Ali, R. L., White, J. M. y Robinson, J. S. (2016). Ethical considerations when researching with pregnant substance users and implications for practice. Addictive Behaviors, 60, 242-243. doi:10.1016/j.addbeh.2016.03.007.

Junta Nacional de Drogas Presidencia de la República Uruguay. (2016). VI Encuesta Nacional en Hogares sobre Consumo de Drogas. Recuperado de https://www. gub.uy/jnd/sites/jnd/files/documentos/publicaciones/201609_VI_encuesta_hogares_OUD_ultima_rev. pdf.

Ko, J. Y., Farr, S. L., Tong, V. T., Creanga, A. A. y Callaghan, W. M. (2015). Prevalence and patterns of marijuana use among pregnant and nonpregnant women of reproductive age. American Journal of Obstetrics and Gynecology, 213, 201.e1-201.e10. doi:10.1016/j.ajog.2015.03.021.

Ley $\mathrm{N}^{\circ}$ 18.256. Protección del Derecho al Medio Ambiente Libre de Humo de Tabaco y su Consumo. Diario Oficial de la República Oriental del Uruguay, Montevideo, Uruguay, 10 de marzo de 2008.

Ley $\mathrm{N}^{\mathrm{0}}$ 19.172. Regulación y Control del Cannabis. Diario Oficial de la República Oriental del Uruguay, Montevideo, Uruguay, 7 de enero de 2014.

Moraes, M., Sosa, C., Umpiérrez, E. y González, G. (2014). Consumo de alcohol, cocaina y cafeína en el embarazo: efectos sobre el embarazo y el niño. Recuperado de http://www. universidad.edu.uy/renderResource/index/resourceId/37211/siteId/12. 
Myers, B., Koen, N., Donald, K. A., Nhapi, R. T., Workman, L., Barnett, W., ... Stein, D. J. (2017). Effect of hazardous alcohol use during pregnancy on growth outcomes at birth: Findings from a South African cohort study. Alcoholism: Clinical and Experimental Research, 42, 369-377. doi:10.1111/acer.13566.

Simón, L., Pastor-Barriuso, R., Boldo, E., Fernández-Cuenca, R., Ortiz, C., Linares, C., ... Galán, I. (2017). SmokeFree Legislation in Spain and Prematurity. Pediatrics, 139, e20162068. doi:10.1542/peds.2016-2068.

Smith, L. M. y Santos, L. S. (2016). Prenatal exposure: The effects of prenatal cocaine and methamphetamine exposure on the developing child. Birth Defects Research
Part C-Embryo Today: Reviews, 108, 142-146. doi:10.1002/ bdrc. 21131.

United Nations Office on Drugs and Crime. (2014). World Drug Report 2014. Recuperado de https://www.unodc. org/.

Valentine, M., Bihm, D. C. J., Wolf, L., Hoyme, H. E., May, P. A., Buckley, D., ... Abdul-Rahman, O. A. (2017). Computer-Aided Recognition of Facial Attributes for Fetal Alcohol Spectrum Disorders. Pediatrics, 140, e20162028. doi:10.1542/peds.2016-2028.

Warner, T. D., Roussos-Ross, D. y Behnke, M. (2014). It's Not Your Mother's Marijuana. Clinics in Perinatology, 41, 877-894. doi:10.1016/j.clp.2014.08.009. 